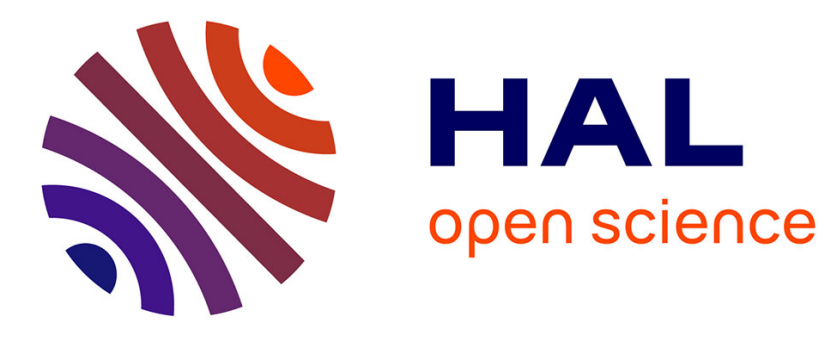

\title{
Enhanced displacement of a liquid pushed by a viscoelastic fluid.
}

J. Avendano, Nicolas Pannacci, Benjamin Herzhaft, Patrick Gateau, Philippe

Coussot

\section{- To cite this version:}

J. Avendano, Nicolas Pannacci, Benjamin Herzhaft, Patrick Gateau, Philippe Coussot. Enhanced displacement of a liquid pushed by a viscoelastic fluid.. Journal of Colloid and Interface Science, 2013, 410 (November), pp.172-180. 10.1016/j.jcis.2013.08.014 . hal-01071696

\section{HAL Id: hal-01071696 \\ https: / hal-ifp.archives-ouvertes.fr/hal-01071696}

Submitted on 6 Oct 2014

HAL is a multi-disciplinary open access archive for the deposit and dissemination of scientific research documents, whether they are published or not. The documents may come from teaching and research institutions in France or abroad, or from public or private research centers.
L'archive ouverte pluridisciplinaire HAL, est destinée au dépôt et à la diffusion de documents scientifiques de niveau recherche, publiés ou non, émanant des établissements d'enseignement et de recherche français ou étrangers, des laboratoires publics ou privés. 
1

\title{
Enhanced displacement of a liquid pushed by a viscoelastic fluid
}

\author{
J. Avendano ${ }^{1,2}$, N. Pannacci ${ }^{1}$, B. Herzhaft ${ }^{1}$, P. Gateau' ${ }^{1}$, P. Coussot ${ }^{3}$ \\ ${ }^{\prime}$ IFP Energies nouvelles, 1 et 4 avenue de Bois-Préau, 92852 Rueil-Malmaison, France \\ ${ }^{2}$ Universidad de los Andes, Laboratorio FIRP, Mérida, Venezuela \\ ${ }^{3}$ Université Paris-Est, Laboratoire Navier (ENPC-IFSTTAR-CNRS), Champs sur Marne, France
}

\begin{abstract}
We consider the displacement, in a rectangular channel, of a Newtonian oil pushed by different types of liquids (Newtonian, shear-thinning, viscoelastic) of slightly higher apparent viscosity. In the absence of viscoelastic effects the interface between the two fluids becomes sharper at larger velocities, so that the thickness of the lateral film left behind increases with the flow rate. On the contrary, with a viscoelastic fluid, the shape of the interface is almost independent of the velocity so that the thickness of the lateral film is approximately constant. Moreover this thickness decreases when the ratio of normal to tangential stresses increases, suggesting that this effect can be attributed to normal stress differences. A heuristic theoretical approach tends to confirm this statement.
\end{abstract}

\section{Introduction}

Chemical EOR (Enhanced Oil recovery) processes consist in the injection of a chemical formulation into the reservoir that will promote the displacement of oil toward the producing well. Different formulations may be used depending on the reservoir characteristics and the technico-economical balance of the project. The polymers commonly used in polymer flooding are synthetic polyacrylamides at concentrations ranging from 200 to 2000 ppm, with high molecular weight - usually around $2010^{6} \mathrm{~g} / \mathrm{mol}$ - and with various degrees of hydrolysation [1]. The rheological behaviour of such polyacrylamide solutions shows two important features: the fluid is extremely shear thinning and exhibits "elastic" properties such as significant normal stress differences or large extensional viscosity. Since the latter effects have been shown to induce spectacular trends under particular flow conditions such as Weissenberg effect (rod climbing), die swell (post extrusion swelling of elastic fluids), apparent filament rigidity, etc, one may suspect that they could have a specific impact on the characteristics of injection flows through porous media. It has for example been observed that the elastic properties of some fluids affect the flow characteristics in other situations where a simple liquid is removed by a viscoelastic fluid: e.g. the elastic properties of the rinsed fluid tend to damp surface disturbances in the flow induced by an impinging jet of a Newtonian liquid [2], the viscoelastic properties affect fingering patterns of the Saffman-Taylor instability [3-5].

In oil recovery, the viscoelasticity of the pushing fluid has been considered through its extensional viscosity [6] which can induce significant increase of flow resistance in a porous medium. Recently it has been suggested that 
the development of significant normal stress differences could be at the origin of enhanced oil recovery [7-12]. This normal stress effect has nevertheless not been demonstrated yet, and the physics at work not understood either: previous studies were indeed carried out in complex geometries and with fluids with poorly controlled rheological properties.

In order to clarify the situation we need to focus on a sufficiently simple situation. In that aim pushing one Newtonian fluid by another one with controlled theological properties in a straight channel seems to be appropriate. The displacement of a more viscous fluid by a less viscous one has been widely studied [13-15]. When viscous effects are sufficiently large compared to capillary effects this leads to the Saffinan-Taylor instability, in which the interface evolves in the form of fingers because this feature minimizes the viscous dissipation. For larger capillary effects a single finger finally forms through the viscous fluid. It was shown that a viscoelastic behavior of the pushed fluid tends to reduce this finger width [16]. This suggests that the opposite effect could occur if the nonviscoelastic fluid is pushed.

With the purpose of examining the specific impact of elastic effects we set up experiments in a simple geometry and with model fluids that have been specially designed to tune independently elastic and viscous components. We focus on flows through a rectangular channel and the displacing fluid is chosen to exhibit normal stress differences but also an apparent viscosity in simple shear very close (but always greater) to that of the displaced fluid. Under these conditions the ideal (displacing) materials would be Boger fluids [5,17-19]. Materials and methods are described first (Section 2), then we present the experimental results (Section 3). In Section 4 we analyze and discuss the trends observed.

\section{Materials and methods}

\subsection{Materials}

The displaced fluid is a Newtonian Silicone oil: Rhodorsil HV-100, of viscosity equal to 0.1 Pa.s. All displacing fluids were immiscible with the displaced fluid. Two families of viscoelastic displacing fluids were formulated. The first one (PAM6000) is an aqueous solution containing a non-ionic Polyacrylamide (Aldrich, $\left.6000 \mathrm{~kg} \cdot \mathrm{mol}^{-1}\right)(0.6 \%$ in weight) and a commercial Newtonian cane syrup (Canadou $\otimes$, called CAN from here) $\left(80 \%\right.$ in weight of sugar) of viscosity equal to $0,16 \mathrm{~Pa} . \mathrm{s}$, with a bactericide $\left(\mathrm{NaN}_{3}\right)(0.05 \%$ in weight). Sodium chloride $(0.2 \%$ in weight) was added to damp possible electrostatic interactions [20] and was found to enhance the reproducibility of data; sodium nitrite was also added and served as a bactericide [21]. The second family is an aqueous solution containing Polyethylene oxide (Aldrich, Molecular Weight: 4000 
kg.mol $\left.{ }^{-1}\right)$ at different concentrations and Polyethylene glycol (Aldrich, $\left.600 \mathrm{~g} / \mathrm{mol}\right)(50 \% \mathrm{in} \mathrm{weight})$. All the fluids were prepared by dissolving the high molecular weight polymer in water (primary solvent), then mixing using a magnetic stirrer at $250 \mathrm{rpm}$ for several hours. Then the secondary solvent (depending on the fluid) was added progressively while mixing with a magnetic stirrer at $400 \mathrm{rpm}$ during 4 hours. In the rest of this paper we will call these PEGPEO followed by the high molecular weight polymer concentration in ppm. Some experiments were carried out with a Newtonian displacing fluid, either with the CAN solution alone, or with a Glycerol in water solution (GLY) at a concentration of $85 \%$ leading to a viscosity of 0.34 Pa.s. A shear-thinning displacing fluid was also used (XAN), i.e. an aqueous solution of Xanthane gum (Aldrich, Xanthonomas campestris) prepared using sodium nitrite as bactericide.

The interfacial tension $(\sigma)$ between the fluids and the displaced silicone oil was measured with a TK 100 KRUSS tensiometer using the Wilhelmy plate technique. We found $34.5 \mathrm{mN} / \mathrm{m}$ for CAN, $26.1 \mathrm{mN} / \mathrm{m}$ for the PAM solutions, and $38.2 \mathrm{mN} / \mathrm{m}$ for the PEO solutions. For some specific tests SPAN 80 (Sigma-Aldrich) was added to the silicone oil in order to obtain two different levels of interfacial tension between the fluids without affecting its viscosity. For $\mathrm{CAN} / \mathrm{silicone}$ oil the interfacial tension was thus lowered to $2.8 \mathrm{mN} / \mathrm{m}$ and for PAM6000/silicone oil it was lowered to $3.1 \mathrm{mN} / \mathrm{m}$.

All the rheometrical tests and the displacement experiments (in the channel) were carried out at room temperature $\left(22 \pm 1^{\circ} \mathrm{C}\right)$.

\subsection{Rheological behavior in simple shear}

The rheological characteristics of these fluids in steady state simple shear, i.e. the shear stress $\tau$ and the first normal stress difference $N_{1}$ (that we will call normal stress in the following) as a function of the shear rate $\dot{\gamma}$, were measured with a controlled stress rheometer (AR2000, TA Instruments) equipped with a cone and plate geometry (diameter: $4 \mathrm{~cm}$; angle: $2^{\circ}$ ) which seems to be an appropriate geometry for measuring the first normal stress difference since the shear rate is homogeneous and the angle is small. From the above data we computed the apparent viscosity: $\eta=\tau / \dot{\gamma}$. The normal force was measured via a gauge placed below the bottom plate. In addition, we have performed oscillatory tests in order to determinate the relaxation time of the viscoelastic formulations using the same geometry. Note that in such a geometry, in a spherical coordinate system $(\theta, \phi, r)$ with $\theta$ the angle from the vertical central axis and $r$ the distance from the central point, we have $\dot{\gamma}=(\sin \theta) d v_{\phi} / d \theta$, $\tau=\sigma_{\theta \phi}$, and $\mathrm{N}_{1}=\sigma_{\theta \theta}-\sigma_{\phi \phi}$, where $v_{\phi}$ is the azimuthal velocity and $\sigma_{i j \in \theta, \phi, r}$ are the stress tensor components [22]. 
After having set up the sample between the tools, the experimental procedure consists to impose first a high shear rate $\left(2000 \mathrm{~s}^{-1}\right)$ during $30 \mathrm{~s}$. Then the sample is left at rest until reaching a stable normal stress value (where the normal stress sensor is set to 0 ) which means that the measured normal stress during flow is taken from this value. The time needed for this stabilization can be up to several hours. The above residual normal stress value can be due to capillary effects associated with the curvature of the free surface of the sample. After that we successively impose steps of constant shear rate $(\dot{\gamma})$ of increasing levels from $0,1 \mathrm{~s}^{-1}$ up to a high value which depends on the fluid but is usually $1000 \mathrm{~s}^{-1}$, then decreasing the shear rate back to the initial value. At each step we record the equilibrium shear stress and first normal stress difference. The difference between the data (apparent viscosity and normal stress) for the increasing and the decreasing ramps was negligible, which means that these materials did not exhibit thixotropic behavior. Only the data associated with the decreasing ramp are presented here. They were shown to be reproducible, even after 30 days. The Reynolds number at the periphery was in any case smaller than 0.5 which means that inertia effects were negligible [23].

In each case it was found that at low shear rates $N_{1}$ fluctuated widely around some value. We considered that these fluctuations result from uncertainties on measurements and/or from artefacts, and we removed the corresponding data. Note that the stress amplitude of these fluctuations is close to the normal stress resolution of the instrument as provided by the supplier for our geometry, i.e. 4 Pa. This does not mean that normal stresses do not exist in that range but that we were able to measure relevant values only at sufficiently high shear rates.

The results of rheological characterization of the different fluid types are shown in Figures 1,2 and 3. The viscosity of PAM solutions is almost constant below some critical shear rate then decreases at higher shear rates. This decrease is stronger for higher concentrations. The normal stress, which is larger for higher concentrations, appears to significantly increase precisely in this range of shear-thinning behavior.

The viscosity of PEGPEO can be considered as constant in our range of shear rates. The normal stress is significant approximately in the same range of shear rates as for the PAM solutions, but it increases more slowly with shear rate. The viscosity and normal stress only slightly increase with the polymer concentration in our range of observation. The apparent viscosity of XAN strongly decreases with shear rate, in contrast with the viscosity of GLY and CAN (see Figure 3). For these materials the normal stress was negligible. 


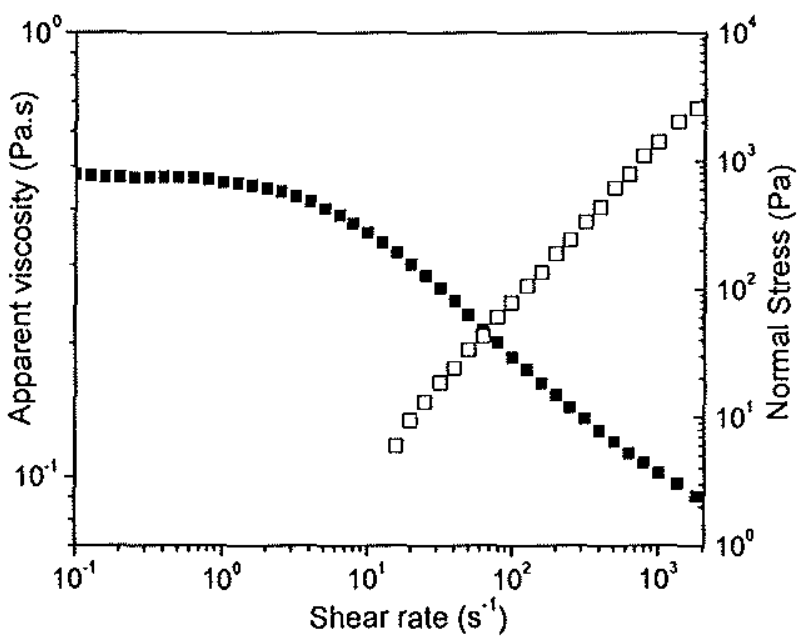

Figure 1: Apparent viscosity (filled squares) and nomal stress (open squares) as a function of shear rate for PAM6000.

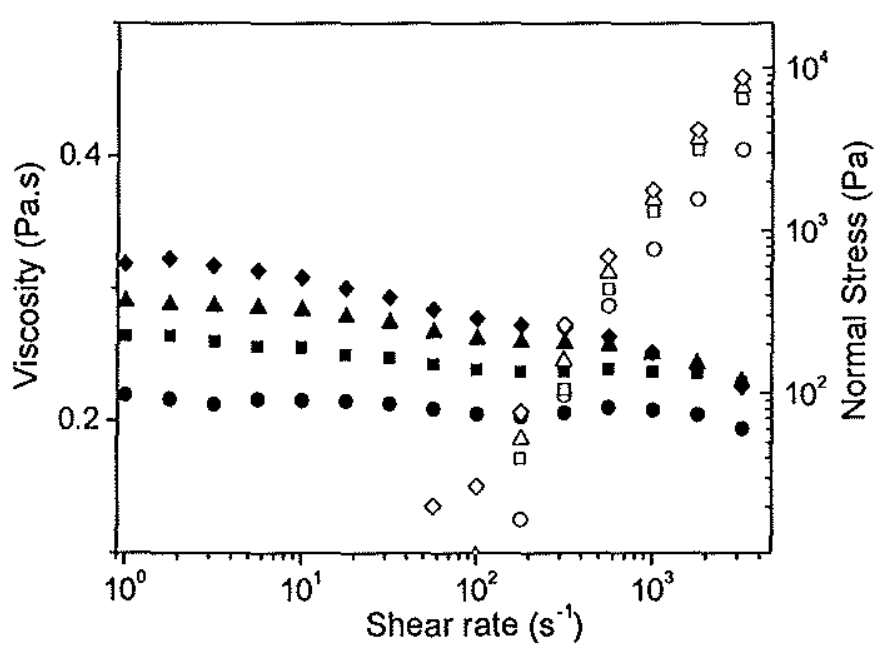

Figure 2: Apparent viscosity (filled symbols) and normal stress (open symbols) as a function of shear rate for PEGPEO at different concentrations (from bottom to top): $500,1500,2000,3000 \mathrm{ppm}$. 


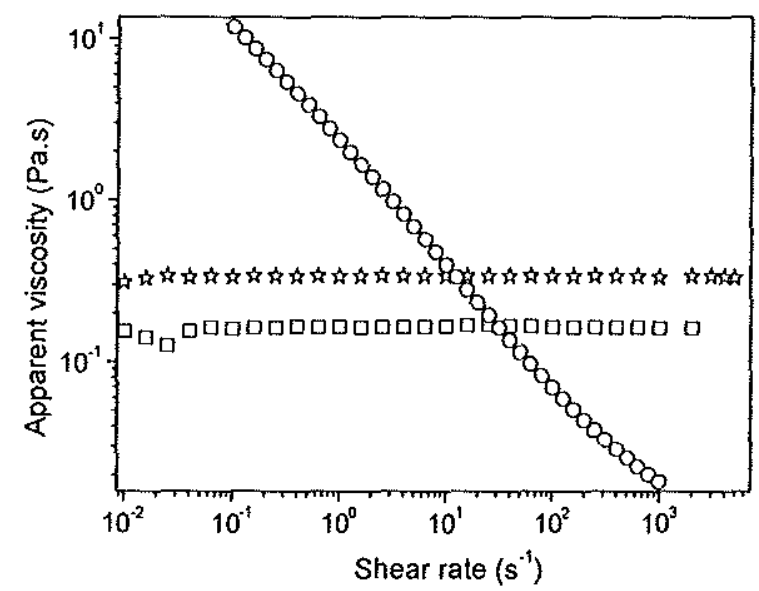

Figure 3: Apparent viscosity of CAN (squares), GLY (stars) and Xanthan solution (circles) as a function of shear rate.

\subsection{Channel flow experiments}

Our experimental set up consists in a cell, i.e. a rectangular cross section channel made of glass (thickness: $b=2 \mathrm{~mm}$; width: $D=2 \mathrm{~cm}$ ) (see Figure 4) through which the Newtonian oil $(\eta=0.1$ Pa.s) is pushed by different types of displacing fluids.
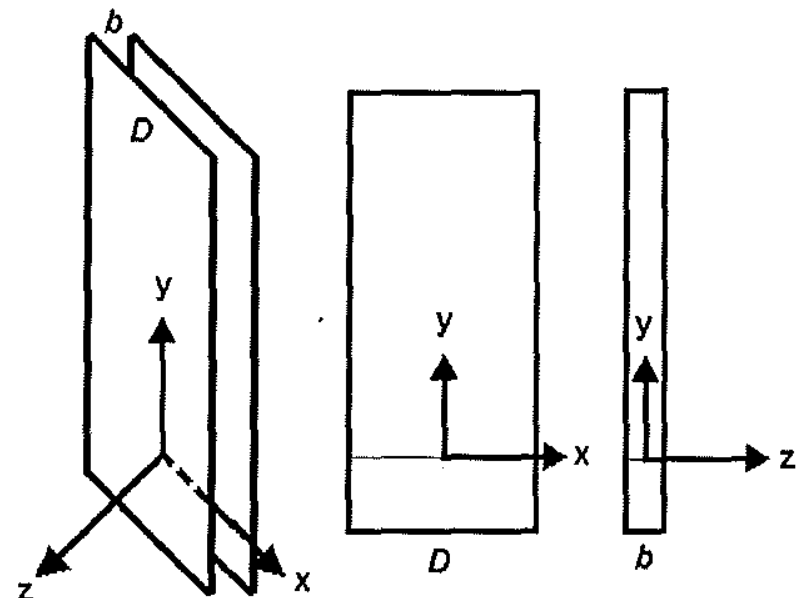

Figure 4: Characteristics of the channel used for the tests: (left) global view of the geometry, (center) view of the widest side, (right) view of the smallest side. 
Before the displacement experiment begins, the channel is partly filled with the displacing fluid (bottom), then the Newtonian oil (displaced fluid) is introduced by the top of the cell and comes into contact with the first fluid so that we do not have remaining air in the cell. The interface between the fluids is slightly concave due to wetting of the aqueous phase on the hydrophilic glass surface. At the beginning of the test the channel is mostly filled with oil (top).

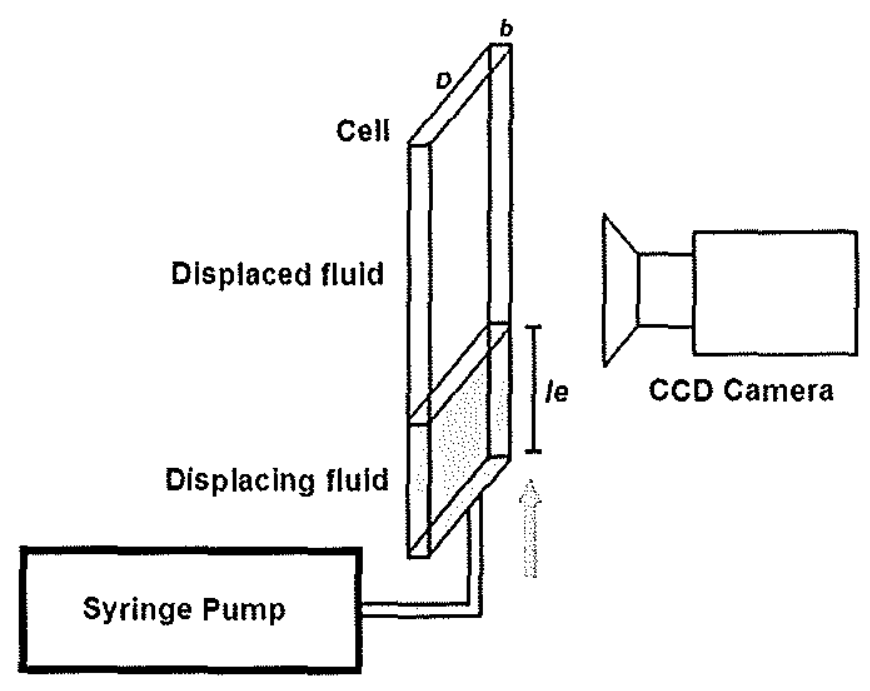

Figure 5: Scheme of the experimental set-up.

After the set up of the two fluids as described above a displacement experiment begins (initial time) with the injection, at a constant flow rate $Q$, of the displacing fluid from the lower inlet using a multiple syringe pump system (KDS220, KD Scientific) which allows us to induce a maximum flow rate of $12 \mathrm{ml} / \mathrm{s}$. This device pushes the oil vertically through the channel (see Figure 5) and induces along the channel an average velocity $V \approx Q / b D$. In the following we will use this velocity as a characteristics of the flow. In our tests a Reynolds number associated with the mean flow characteristics could be computed from the following expression: $\operatorname{Re}=\rho V b / \eta$, in which $\rho$ is the fluid density. In this expression, for a non-Newtonian fluid we used the value of the apparent viscosity of this fluid for the apparent sliear rate of the flow $(\bar{\gamma})$ defined in Section 4.1. In our tests this Reynolds number was always smaller than 1 , which indicates that the flow was always laminar.

The flow characteristics are observed from the initial time via the evolution of the apparent interface from above the largest channel side (plane $(x, y)$ ). The images are acquired with a CCD Camera monochrome CV-M10 $50 \mathrm{~Hz}$ equipped with a macro-objective. The illumination was obtained thanks to a fibre optic illuminator behind 
the cell with a "white screen". The images were processed with MATLAB in order to obtain the interface profiles (see example in Figure 6). Note that this is only an apparent interface that we can observe with this technique. The effective interface is likely curved along the z-axis, so that the observed shape might correspond to the position of the front line of the interface along the y-axis, in other words the intersection of the interface and the plane $\mathrm{z}=0$. In the following we will refer to this line as the "interface". The reproducibility of the whole flow process and interface image processing was found to be excellent: two interface profiles obtained from two different tests under the same experimental conditions cannot be distinguished from each other. The maximum time of acquisition of the camera was $20 \mathrm{~ms}$ which means that the maximum displacement in the small duct at the highest velocity $(10 \mathrm{~cm} / \mathrm{s})$ was $6 \mathrm{~mm}$.

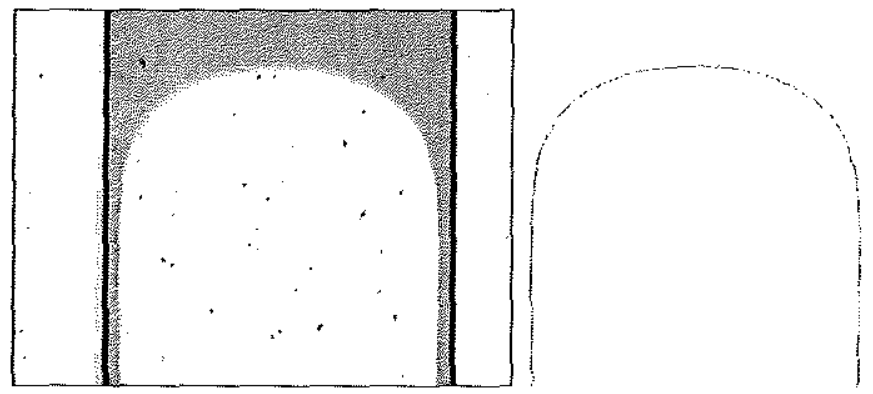

Figure 6: Typical interface profile (right) obtained after image processing of a basic picture (left).

\section{Experimentals results}

\subsection{Flow regimes}

During the propagation of the interface from the initial time, two stages can be observed in the displacement experience. We have first a transient stage, over the very first centimetres of displacement, in which the interface evolves from a slightly concave shape to a convex one (see Figure 7). Then a second stage is observed during which the shape of the interface remains apparently constant all along the channel. The distance needed to reach this regime does not seem to depend significantly on the flow conditions and material type (see Figure 7). A typical evolution of the interface profile from the initial time after image processing is shown in Figure 9 which confirms this description of the flow evolution in two stages. Furthermore the interface profiles measured over a length of 1 $\mathrm{cm}$ along the channel axis in the second regime are effectively identical over a significant flow distance (at least 20 $\mathrm{cm}$, i.e. 10 times the channel width and 100 times the channel thickness) (see Figures 7 and 8 ), which means that the characteristics at the flow front are stationary. By the way we can remark that these profiles are unambiguously different for two different displacing fluids (see Figure 7). From now on we will use the stable front shape curve as a characteristic of the displacement process. 
Figure 7: Successive views of the interface profile in the plane $(x, y)$ for CAN (left of each column block) and PAM-6000 (right of each column block) at different times: (from bottom to top of left then righ column block) 0 , $0.3,0.5,0.6,0.8,1,1.5,2 \mathrm{~s}$. The injection velocity of $10 \mathrm{~cm} / \mathrm{s}$ is constant but due to the slower resulting flow rate during the start up regime the first six pictures correspond to $0 \mathrm{~cm}<y<5 \mathrm{~cm}$ while the two last ones correspond to $5 \mathrm{~cm}<y<10 \mathrm{~cm}$ and $10 \mathrm{~cm}<y<15 \mathrm{~cm}$. 


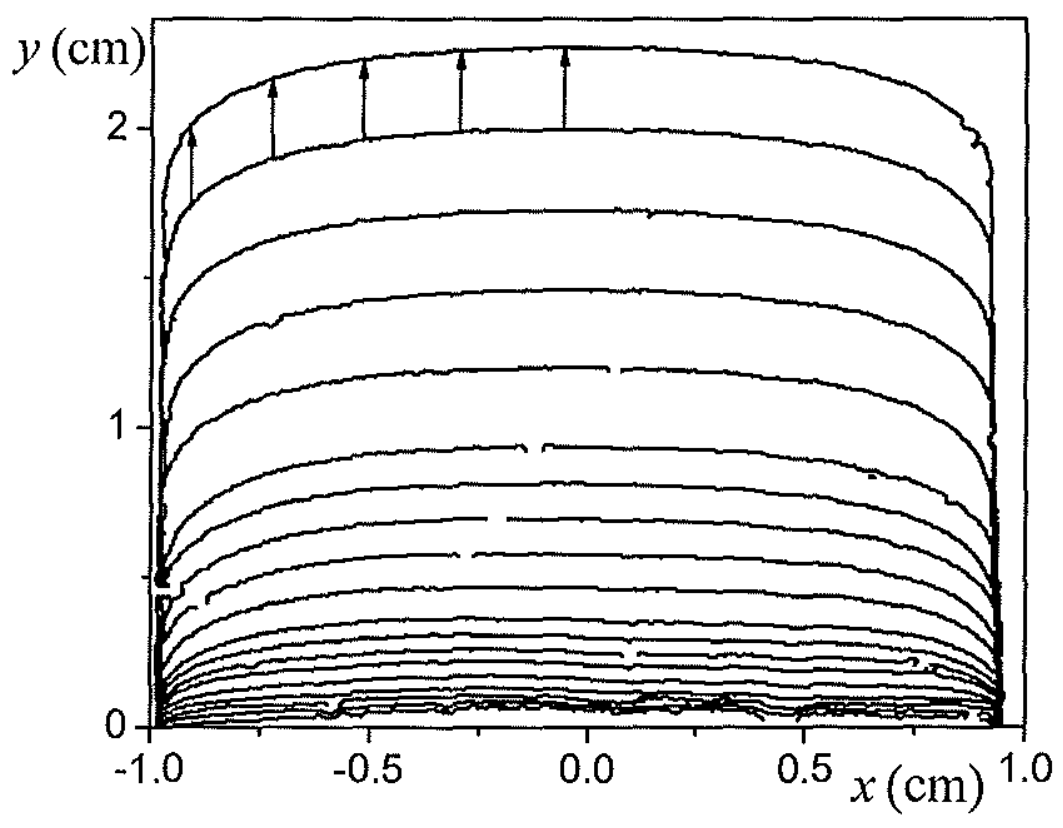

Figure 8: Successive views (every $250 \mathrm{~ms}$ ) of the interface profile in the plane $(x, y)$ for CAN for a flow at 1 $\mathrm{cm} / \mathrm{s}$ from the initial time of injection. The (identical) arrows between the two last profiles show that their shape around the front is identical.

\subsection{Impact of velocity}

For all displacing fluids the interface shape is almost flat at low velocities, then the curvature increases as the velocity increases (see Figure 9). For a Newtonian displacing fluid the interface shape significantly evolves with the velocity increase, it becomes sharper so that the thickness of the layer left along the channel side reaches large values. Beyond some critical velocity (say around $20 \mathrm{~cm} / \mathrm{s}$ ) the interface seems to be unstable, its shape is uneven and the reproducibility is poor. For a viscoelastic fluid the interface profile rapidly reaches an almost constant shape when the velocity increases, and no instability is observed in our range of velocities. 


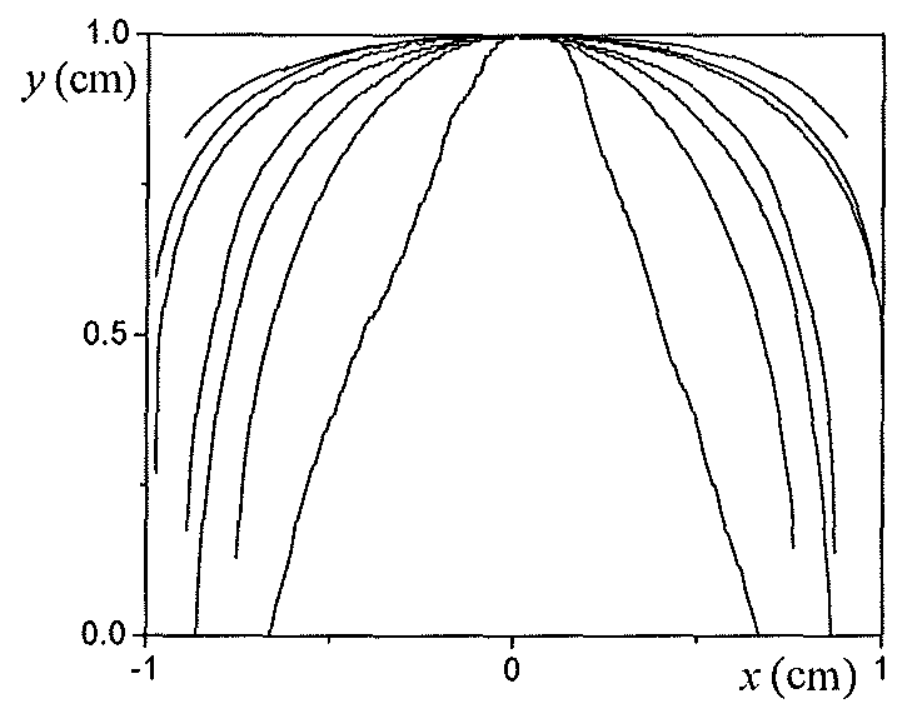

(a)

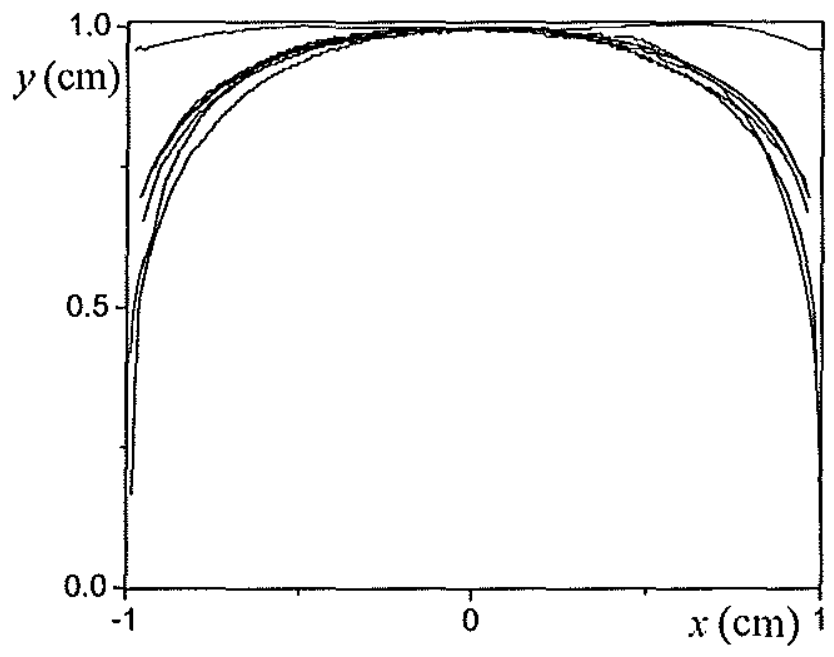

(b)

Figure 9: Interface profile at different velocities for CAN (a): (from top to bottom) $0.2,0.5,1,5,8,15,20$ $\mathrm{cm} / \mathrm{s}$; and PAM6000 (b): $0.1,0.5,1,5,8,15,20 \mathrm{~cm} / \mathrm{s}$.

\subsection{Impact of the viscosity of the displacing fluid}

In order to be able to separate the impact of viscoelastic effects from those due to the viscosity of the displacing fluid we carried out experiments with two Newtonian displacing fluids of different viscosities (by a factor 2) and a 
shear-thinning displacing fluid (i.e. the Xanthan solution). We observe that the interface profiles do not significantly depend on the viscosity of the displacing fluid and, more spectacularly, are also the same with a displacing fluid with a shear-rate dependent viscosity (see Figure 10).

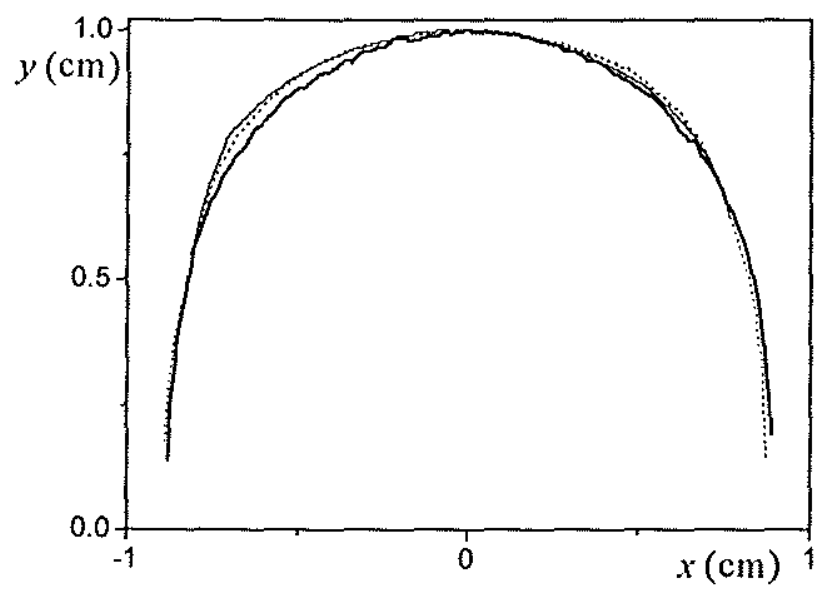

Figure 10: Interface profile at different velocities for CAN (dotted line), GLY (thick line) and XAN (thin line) displacing Silicone oil at $5 \mathrm{~cm} / \mathrm{s}$.

\subsection{Impact of surface tension}

In order to be able to separate the impact of viscoelastic effects from those due to surface tension we carried out experiments at a much lower interfacial tension. The results are shown in Figure 11 for CAN but similar trends were obtained for PAM6000. It appears that the interface profile does not seem to significantly depend on the surface tension. Some slight difference can be remarked but the critical point is that this difference does not evolve in a specific way for increasing velocity and finally the global evolution over our velocity range is similar for both fluids. This suggests that there is no clear tendency concerning the difference obtained for each velocity, this is more like some fluctuations. This leads us to conclude at the overall similarity between the two set of data. 


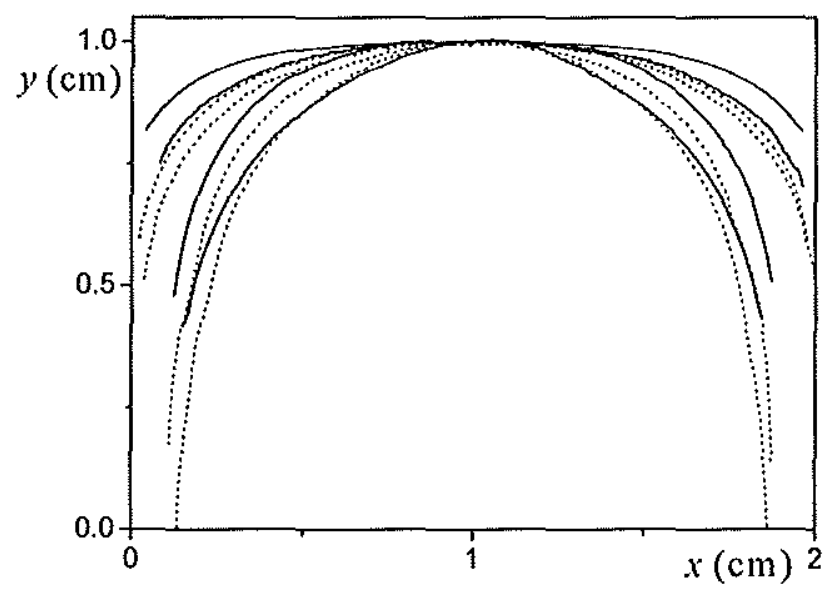

Figure 11: Interface profiles at different velocities for CAN displacing Silicone oil at high $(34.5 \mathrm{mN} / \mathrm{m})$ (dotted line) or low $(3.1 \mathrm{mN} / \mathrm{m}$ ) (continuous line) surface tension: (from top to bottom) $0.5,1,5,8 \mathrm{~cm} / \mathrm{s}$.

\subsection{Impact of polymer concentration}

In the case of PEGPEO we could vary the polymer concentration and observe the impact on the interface profile. In that case, for a given velocity the interface profile becomes flatter as the polymer concentration increases (see Figure 12).

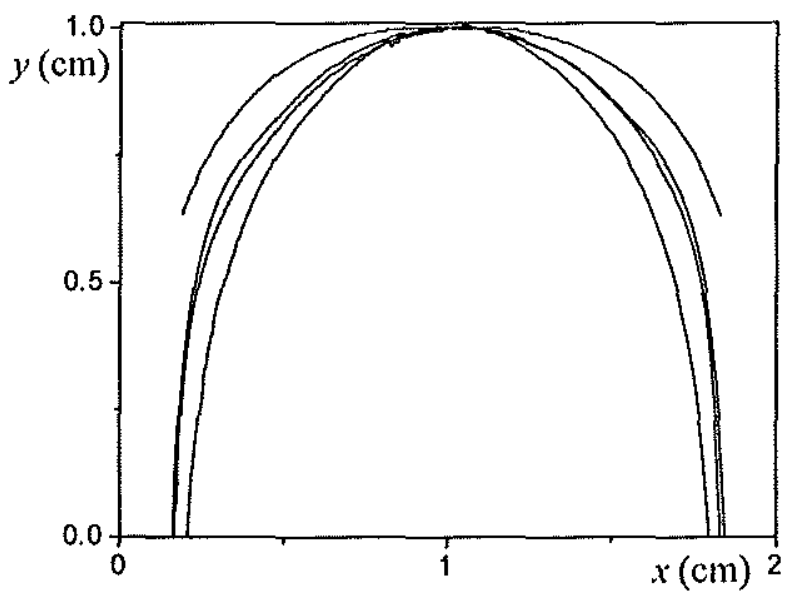

Figure 12: Interface profiles at a velocity of $5 \mathrm{~cm} / \mathrm{s}$ for PEGPEO at different concentrations: (from top to bottom): $3000,2000,1500,500 \mathrm{ppm}$. 


\section{Analysis and discussion}

Let us start by reviewing our main experimental observations:

- For all fluid types the front of the interface profile, say over a distance of the order of the channel width, keeps the same shape all along the channel.

- For simple liquids (Newtonian or shear-thinning) the interface profile becomes sharper for increasing velocity; this shape does not depend on the viscosity of the displacing fluid and is not affected by a shear-thinning behavior.

- For our polymer solutions the interface profile is constant beyond some critical (low) velocity; with regards to the Newtonian case the profile is enlarged as if the interface was pushed towards the channel sides by some effect. This effect is stronger for increasing polymer concentration.

- There does not seem to be a significant effect of interfacial tension on the shape of the interface.

Now we attempt to analyze the flow characteristics with regards to the observed trends. A complete approach via numerical simulation might be carried out, with the difficulty to describe transient flows with a thin layer along the boundaries and to take into account complex rheological behavior (including in particular normal stresses). Instead we will here discuss of the different characteristics of the problem with regards to theoretical knowledge in simple cases and by this way try to find out the origin of the observed effects. Our starting point, which will then constitutes a situation of reference, is the steady state flow of a single Newtonian fluid through a channel. Afterwards we will discuss essentially through a qualitative approach the flow characteristics of a two-phase flow with regards to this well controlled situation.

\subsection{Characteristics of a uniform flow of a single Newtonian fluid through a rectangular channel}

We consider the uniform, steady state, laminar flow of a Newtonian fluid of viscosity $\mu_{0}$ through a channel of the fype described in Figure 4, with $b<<D$. In a plane $(y, z)$ far from the channel sides, at first order the velocity is independent of $x$ and the only non-zero component $(y)$ of the velocity is along the $y$ axis. Locally we thus have essentially a simple shear with a shear rate $\dot{\gamma}=d v / d z$. Under these conditions the deviatoric part of the stress tensor does not contain diagonal components $\left(\tau_{y y^{\prime}}=0=\tau_{z z}\right)$, the remaining component $\left(\tau_{y z}\right)$ only depends on $\dot{\gamma}$, and the momentum equation reduces to 
$\frac{\partial \tau_{y z}}{\partial z}-\frac{\partial p}{\partial y}=0 ;-\frac{\partial p}{\partial z}=0$

in which $p$ is the pressure. For a Newtonian fluid we have $\tau_{y z}=\mu_{0} \dot{\gamma}$ and the momentum equation leads to $\partial \tau_{y_{2}} / \partial z=A=\partial p / \partial y$, in which $A<0$ is a constant. We deduce the usual « Poiseuille » velocity profile:

$v(z)=\frac{\nabla p}{2 \mu_{0}}\left(z^{2}-\frac{b^{2}}{4}\right)$

in which $\nabla p=A$. In that case the local shear rate expresses as $\dot{\gamma}=\left(\nabla p / \mu_{0}\right) z$. Thus the shear rate amplitude at the wall is $\dot{\gamma}_{m}=\left(\nabla p / \mu_{0}\right) b / 2$ and the mean shear rate $\bar{\gamma}=\left(\nabla p / \mu_{0}\right) b / 4$. It is useful to notice that the latter expression may be related to the average velocity $V=1 / b \int_{-b / 2}^{+b / 2} v d z=\left(\nabla p / \mu_{0}\right) b^{2} / 12$ :

$\overline{\dot{\gamma}}=\dot{\gamma}_{m} / 2=3 \mathrm{~V} / \mathrm{b}$

For such a uniform flow a fictive line initially perpendicular to the flow direction is progressively elongated as the fluid advances. In addition, since the local velocity is proportional to the average velocity, the deformations induced by the flow at different velocities are similar after the same average displacement of the fluid.

At the approach of the lateral walls $(x= \pm D / 2)$ the velocity field differs from that given by equation (2). For a stable flow there is no secondary flow so that we still have a simple shear with only one non-zero component of the velocity along $y$ but now depending on both $x$ and $z$. Gondret [24] provided the exact expression of this velocity profile $v(x, z)$. Here we directly show the resulting deformations of an initially straight line according to this expression (see Figure 13). This velocity field is again proportional to the average velocity, as expected for a Stokes flow. As a consequence a fictive line initially perpendicular to the flow direction is progressively elongated as the fluid advances, and if we withdraw two successive profiles obtained for this line we get the basic velocity profile (see Figure 13).

Moreover this velocity profile is such that $v( \pm D / 2, z)=0$, which leads to some perturbation (with regards to the flow far from the edges) affecting the flow characteristics up to a distance of the order of $b$, beyond which the velocity profile (2) is recovered. As a consequence the velocity along $z=0$ is uniform between $x \approx(D / 2)-b$ and $x \approx b-(D / 2)$. It follows that roughly speaking the shear rate along the channel sides and in the plane $(x, y)$ is in the same order as the shear rate far from the channel sides and in the plane $(y, z)$, since in both cases the velocity varies from 0 at the wall to $V$ at a distance of the order of $b$.

Thus, for a Newtonian fluid flowing through such a rectangular channel we can conclude that the velocity profiles observed in the transversal plane (namely $(x, y)$ ) have some qualitative characteristics similar to those which would be observed in a longitudinal cross-section $(y, z)$ far from the channel sides: the deformation induced is 
independent of the velocity and the shape of the profiles at the approach of the walls are roughly similar. This result is natural considering that the fluids are sheared at the same time along $z$ as a result of the presence of the bottom and top plates and along $x$ as a result of the presence of the side edges. This suggests that for other fluid types and maybe for two-phase flows we can directly look at the shape of the interface in the transversal plane $(x, y)$ as reflecting the general evolution of the interface between the two fluids in all directions.

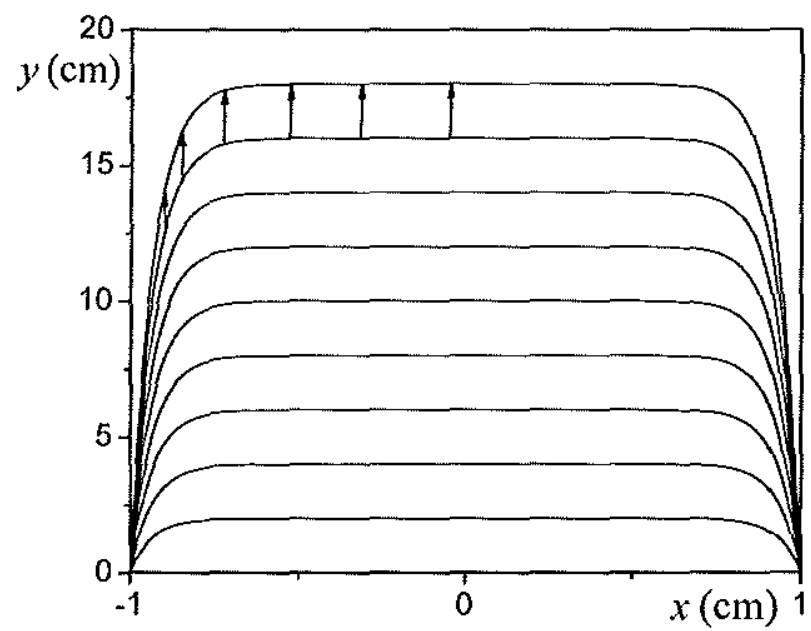

Figure 13: Successive interface profiles for a Newtonian fluid flowing through our channel according to Gondret [26]. The (identical) arrows illustrate the shift between two successive profiles.

\subsection{Characteristics of two-phase flows}

Let us consider the case of a Newtonian fluid pushing another Newtonian fluid (of smaller viscosity) through a rectangular channel. We first assume that surface tension effects are negligible, which implies that only viscous effects should play a role on the flow characteristics of both the displacing and displaced fluids. For a Newtonian fluid pushing another Newtonian fluid of different viscosity, as the interface between the two fluids is deformed, the pressure gradient cannot be uniform in each cross-section of the channel (as predicted by the momentum equation for the flow of a single fluid (see above)). This means that the interface shape has to adjust somewhat to take into account that effect, and differs from that obtained for a monophasic flow (i.e. two fluids of similar viscosity). However for two Newtonian fluids with dominant viscous effects, due to the linearity of the problem, we should get a similar deformation after the same displacement of fluid whatever the velocity. 
Let us recall that the interface profiles describe the deformation of the front of the interface resulting from the velocity field. Subtracting one interface profile from the subsequent one thus provides the velocity profile of the front of the interface. Under these conditions a critical point is that our general observation (for any type of fluid) of an apparently constant interface profile leads to a velocity profile equal to a plateau at least up to a distance of the order of $0.2 \mathrm{~mm}$ from the channel sides (see Figure 9). For a Newtonian fluid this result clearly contrasts with the above theoretical predictions of a progressively elongated interface profile and a shearing up to several millimeters for a monophasic flow. This suggests that for a two-phase flow there is some effect which tends to stabilize the interface shape. Note that this apparent stationarity of the interface beyond some distance implies that its shape is decided during the initial transient stage, i.e. the start up flow (see Figure 8).

Moreover we have seen that for two Newtonian fluids, if viscous effects are dominant we should get similar interface profiles for different velocities. This is clearly not the case (see Figure 9a), which implies that there is also some effect playing a role in the development of the stationary interface.

These remarks, i.e. stabilization of the interface shape after some distance and variation of this shape with the velocity, finally strongly suggest that surface tension effects play a significant role in the process at least for Newtonian fluids. The relative importance of viscous and capillary effects may be appreciated from the value of the Capillary number, i.e. $C a=\eta V / \sigma$. In our tests $C a$ is generally situated between 0.1 and 1 , except for experiments using low interfacial tension fluids. This means that a priori capillary effects should play a significant role in most cases and even sometimes govern the flow characteristics along the interface. More generally in that frame of description we expect that the shape of the interface should be entirely governed by the value of the Capillary number.

In order to have a simpler way of comparison of the different stationary profiles we focus on a single parameter which makes it possible to appreciate the shape of these profiles: the thickness of the remaining layer of displaced liquid at some distance behind the front. In the above figures we focused on the velocity profiles over a distance of $1 \mathrm{~cm}$ from the front of the interface. The thickness of the remaining displaced fluid along the channel sides as deduced from our pictures in the plane $(x, y)$ then very slowly varies with the distance from the front. Here we will arbitrarily consider that the apparent thickness at a distance of $4 \mathrm{~cm}$ from the front of the interface is the typical thickness $(e)$ of the film left behind the flow of the displacing fluid, but we have in fact no information about the thickness over a much longer distance from the front. In Figure 14 this thickness is represented as a function of the Capillary number (at a power $2 / 3$ in the spirit of Bretherton approach (see below)). At first sight it appears that for a Newtonian or shear-thinning displacing fluid this thickness is much higher than for a viscoelastic fluid for the same Capillary number. Such a result is consistent with our above observations (see Section 3.5) of the shape of the interface profile over a distance 
of $1 \mathrm{~cm}$ for the different fluid types, since a sharper interface in this region leads to a larger thickness of remaining displaced fluid at some distance behind.

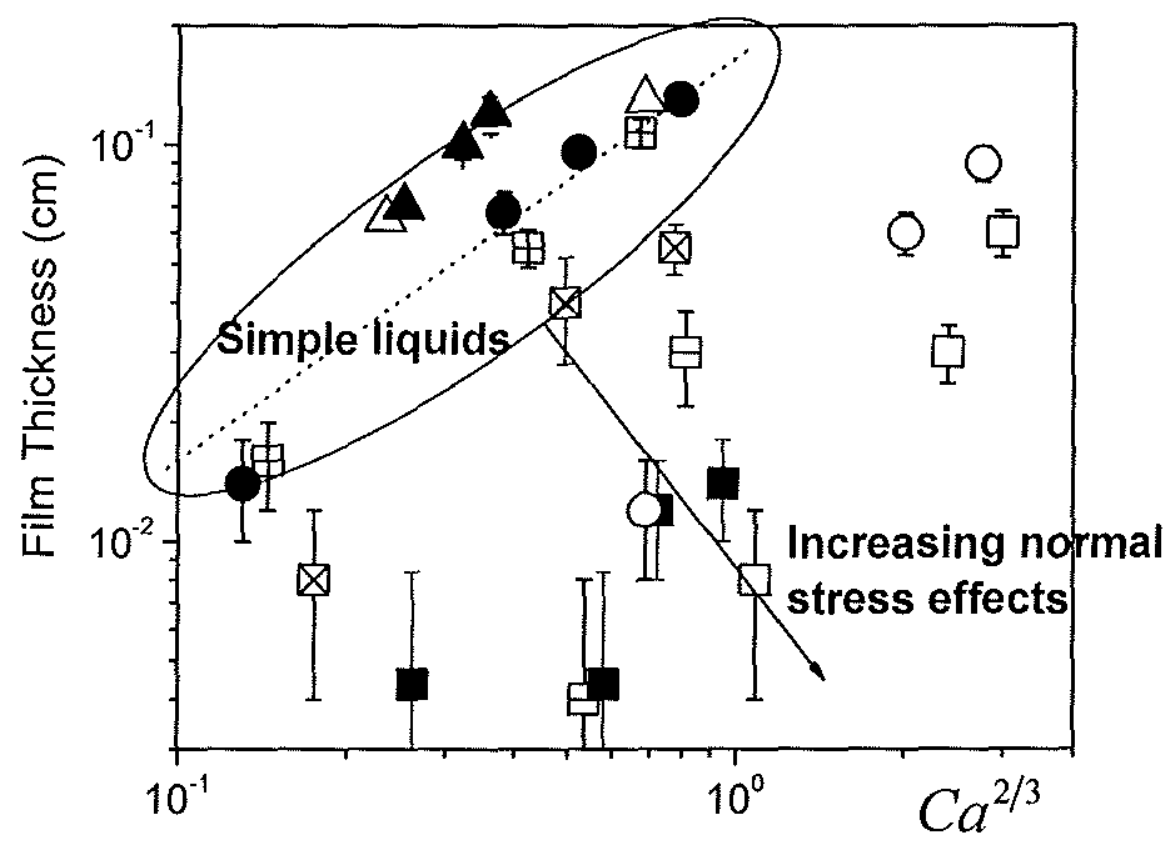

Figure 14: Lateral film thickness (see text) as a function the Capillary number for CAN (circles), PAM6000 (squares), XAN (triangles) and GLY (stars) at standard (filled symbols) and low (open symbols) interfacial tension, and for PEGPEO at different concentrations: $500 \mathrm{ppm}$ (vertical cross-squares), $2000 \mathrm{ppm}$ (diagonal cross-squares) $3000 \mathrm{ppm}$ (squares with horizontal line). The dotted line has a slope 1.

More precisely it seems that we have indeed a flow mostly governed by the Capillary number for simple liquids such a CAN, GLY and XAN for which $e(\mathrm{Ca})$ seems to follow a single straight line (of slope 1) (see Figure 14). The different positions of this line for the different fluids might be due to the fact that in the expression of $\mathrm{Ca}$ we only take into account the viscosity of the displacing fluid but for two fluids of different viscosities the ratio of these viscosities should also play a role (as explained above for two fluids in the absence of capillary effects). This ratio significantly differs from 1 for GLY and XAN. Finally these results tend to show that we could simply expect a dependence of the shape of the interface on $\mathrm{Ca}$ and the viscosity ratio. 
This situation is reminiscent of the analysis of Bretherton [25] who predicted the thickness of the liquid layer left along the walls of a duct initially filled with liquid and through which a bubble penetrates. This analysis leads to $e=l \mathrm{Ca}^{2 / 3}$, in which $l$ is half the size of the bubble perpendicularly to the flow direction [26]. Despite the fact that in his case the viscosity ratio was close to 0 , a very different situation from ours, there is a good agreement of the Bretherton law with our data for simple liquids in terms of the variations of the thickness which indeed appears to be approximately proportional to $\mathrm{Ca}^{2 / 3}$ (see Figure 14). For CAN the coefficient of proportionality $(l=0.22 \mathrm{~cm}$ ) is even close to the channel thickness, as predicted by Bretherton.

However the things seem more complex when one looks at the data for a low interfacial tension (see Figure 14). Although only the interfacial tension has been changed the data for CAN do not at all collapse along those obtained at standard surface tension, in agreement with the observations (see Section 3) showing roughly similar interface shapes in both cases. We have here an unexpected and unexplained effect. The point is that, as we will see below we get a consistent interpretation of all the trends except that one. So we suspect that, for some reason, the effective interfacial tension during the process was not that found from independent measurements.

Leaving apart this problem we can further use the representation of data in terms of the layer thickness as a function of the Capillary number to understand the origin of the flow characteristics for the viscoelastic fluids (see Figure 14). We can remark that the data for PAM6000, if displaced towards low $\mathrm{Ca}$, may approximately fall along the curve obtained for CAN (at standard surface tension). This shifting could be obtained by using a much higher (fictive) surface tension for PAM6000. This suggests that viscoelastic effects could induce effects similar to an increase of surface tension. This analysis is confirmed by the fact that the data for PAM6000 at low surface tension approximately collapse along the same curve as data with standard surface tension: since viscoelastic effects play now a dominant role as a fictive surface tension, lowering the effective surface tension has a negligible impact on the flow characteristics.

Our conclusion is further supported by the trends observed for PEGPEO solutions: as their concentration is increased the data for $e$ vs $\mathrm{Ca}^{2 / 3}$ appear to move from the region of simple liquids (see Figure 14) to the region where viscoclastic effects are dominant.

\subsection{Heuristic approach}

Here we attempt to provide some heuristic explanation of the observed trends. For the channelized flow under consideration there can be some residual lateral motion, in particular associated with the slight expansion of the interface between the two fluids towards the channel sides, but the predominant motion is along the channel axis. This implies that we are essentially dealing with a simple shear and elongational effects are negligible. Under these 
conditions the differences in interface shape observed between the viscoelastic systems and the Newtonian liquids of similar apparent viscosity can only be explained by their rheological differences in simple shear flows, namely the existence of significant normal stresses. This effect appears to be stronger for increasing normal stress difference and it seems that it prevails over interfacial effects. The importance of normal stress effects can be appreciated from the ratio of normal to tangential stress. It is particularly interesting to remark that for our different materials, this ratio increases from very low values to much more than 1 as we use solutions of PEGPEO at increasing concentration then a solution of PAM6000 (see Figure 15). This increase occurs more or less at the same rate as the data for $e$ vs $C a^{2 / 3}$ are displaced from the simple liquid region to the viscoelastic region in Figure 14.

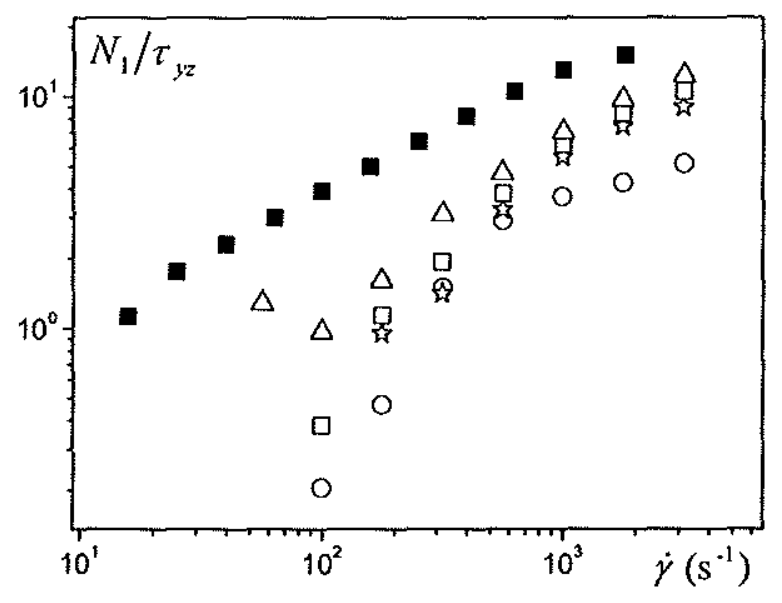

Figure 15: Ratio of the normal to the shear stress as a function of the shear rate for PAM6000 (filled squares) and for PEGPEO at different concentrations: (circles) 500, (stars) 1500, (open squares) 2000, (triangles) 3000 ppm.

Let us try to have some ideas of the origin of this effect by considering again the case of a uniform steady flow through a channel without side effects, but now for a viscoelastic fluid exhibiting normal stresses. For the sake of simplicity we represent the steady state simple shear behavior of this viscoelastic fluid with linear expressions for the shear and normal stresses: $\tau_{y z}=\eta \dot{\gamma}, \tau_{z z}=\alpha \dot{\gamma}$, in which $\alpha$ is a viscosity coefficient for the normal stress. Here we only consider the flow in the region of positive $z$ values. Note that this model represents very well the behavior of the PEGPEO (see Section 2) but is a rough approximation in the case of the PAM for which one would need to assume $\eta$ and $\alpha$ to be apparent viscosities varying with $\dot{\gamma}$. Since $\tau_{z z}$ only depends on $z$ (uniform flow) the momentum equation implies that the pressure expresses as 
$p=f(y)+g(z)$. Finally we deduce $g(z)=A z$ with $\partial \tau_{y z} / \hat{\partial z}=A$, so that the velocity profile is still given by the above Poiseuille law. However the pressure is now given as:

$$
p(y, z)=A y+\alpha \dot{\gamma}=A(y+\alpha z / \eta)
$$

in which the additional constant was dropped.

Thus when the fluid developing normal stresses is alone in the channel its flow characteristics are similar to those of a simple Newtonian fluid except that the pressure distribution is more complex. When it is in contact with a Newtonian fluid of similar apparent viscosity but for which the pressure simply varies linearly with $y$ the interface does not simply result from the fluid deformation according to Poiseuille law, since it would not allow for the pressure continuity (expected in the absence of surface tension effects). The interface shape will tend to adjust in order to take into account the pressure shift due to the normal stress effect: according to the above equation the interface will be shifted towards larger $y$ (where the pressure is smaller) for larger $z$.

If, considering that the effects induced in this longitudinal cross-section will induce similar effects in the plane $(x, y)$, we extrapolate the above results to the flow characteristics in the transversal direction and we find that the interface profile will be significantly affected if the second term of the pressure expression is of the order of the first term for the start up distance $L$. Since apparently we have $L \approx D$ (see Figure 8 ). The importance of this effect will be expressed by the dimensionless number $\alpha / \eta$, or more generally the ratio of the normal stress to the shear stress in simple shear: $N_{1} / \tau_{y z}$.

Let us now compare the prediction of this analysis with our data. For our channelized flows the maximum shear rate $\left(\dot{\gamma}_{m}\right)$ ranges from $30 \mathrm{~s}^{-1}$ to $600 \mathrm{~s}^{-1}$ when the velocity ranges from $1 \mathrm{~cm} \cdot \mathrm{s}^{-1}$ to $20 \mathrm{~cm} \cdot \mathrm{s}^{-1}$, which roughly corresponds to the region for which a normal stress could be recorded (see Figure 15). Moreover in this range of shear rates, for PAM6000 $N_{1} / \tau_{y z}$ is larger than 1 and increases for increasing shear rate (see Figure 15), which according to the above approach means that the normal stress plays a major role and could be at the origin of the effect observed in the fluid-fluid displacement experiment. In this regime there might be an effect of saturation of the impact of normal stress beyond a value of 1 for $N_{1} / \tau_{y z}$, which could explain the fact that the interface profile no longer changes in that range (when the velocity increases). For the PEGPEO solutions $N_{\mathrm{b}} / \tau_{y z}$ becomes larger than 1 for a sufficiently high shear rate, which is consistent with the observation that at a given velocity the interface shape becomes wider for increasing concentration (see Figure 12). 


\section{Conclusion}

We carried experiments in which a simple liquid was displaced by different types of fluids. These experiments made it possible to prove that viscoelastic fluids exhibiting normal stress differences tend to push the simple liquid with a less curved profile of the interface so that less fluid is left behind along the channel sides. Our discussion demonstrated that this effect in fact finds its origin in the start up flow conditions, i.e. the shape of the interface is determined during the first stage of the flow. Nevertheless a heuristic approach shows that we can expect a predominance of normal stress effects if the ratio of the normal stress to shear stress is sufficiently large, which is the case for our viscoelastic fluids. A good qualitative agreement was found between this approach and the experimental data. However two-phase flows of complex fluids constitute a very difficult field which could gain from numerical simulations.

\section{References}

[1] A. Ait-Kadi, P.J. Carreau, G. Chauveteau, Rheological Properties of Partially Hydrolyzed Polyacrylamide Solutions, Joumal of Rheology. 31 (1987) 537-561.

[2] T.T. Hsu, T.W. Walker, C.W. Frank, G.G. Fuller, Role of fluid elasticity on the dynamics of rinsing flow by an impinging jet, Physics of Fluids. 23 (2011) 033101-033101-10.

[3] J.. Ro, G.. Homsy, Viscoelastic free surface flows: thin film hydrodynamics of Hele-Shaw and dip coating flows, Journal of Non-Newtonian Fluid Mechanics. 57 (1995) 203-225.

[4] D. Bonn, J. Meunier, Viscoelastic Free-Boundary Problems: Non-Newtonian Viscosity vs Normal Stress Effects, Physical Review Letters. 79 (1997) 2662-2665.

[5] E.F. Quintella, P.R. Souza Mendes, M.S. Carvalho, Displacement flows of dilute polymer solutions in capillaries, Journal of Non-Newtonian Fluid Mechanics. 147 (2007) 117-128.

[6] D.M. Jones, K. Walters, Extensional Viscosity Effects in EOR, SPE Annual Technical Conference and Exhibition, 2-5 October 1988, Houston, Texas, Ed.: Society of Petroleum Engineers ISBN: 978-1-55563-578-7

[7] J. Hou, Z. Liu, S. Zhang, X. Yue, J. Yang, The role of viscoelasticity of alkali/surfactant/polymer solutions in enhanced oil recovery, Journal of Petroleum Science and Engineering. 47 (2005) 219-235.

[8] W. Demin, C. Jiecheng, Y. Qingyan, G. Wenchao, L. Qun, C. Fuming, Viscous-Elastic Polymer Can Increase Microscale Displacement Efficiency in Cores, SPE Annual Technical Conference and Exhibition, 2-5 October 1988, Houston, Texas, Ed.: Society of Petroleum Engineers ISBN: 978-1-55563-910-5.

[9] X. Huifen, J. Ye, K. Fanshun, W. Junzheng, Effect of Elastic Behavior of HPAM Solutions on Displacement Efficiency under Mixed Wettability Conditions, SPE Asia Pacific Oil and Gas Conference and Exhibition, 1820 October 2004, Perth, Australia, ISBN: 978-1-55563-979-2. 
[10] D. Wang, G. Wang, W. Wu, H. Xia, H. Yin, The Influence of Viscoelasticity on Displacement Efficiency-From Micro- to Macroscale, SPE Annual Technical Conference and Exhibition, 11-14 November 2007, Anaheim, California, U.S.A, ISBN: 978-1-55563-148-2.

[11] H. Jiang, W. Wu, D. Wang, Y. Zeng, S. Zhao, J. Nie, The Effect of Elasticity on Displacement Efficiency in the Lab and Results of High Concentration Polymer Flooding in the Field, SPE Annual Technical Conference and Exhibition, 21-24 September 2008, Denver, Colorado, USA, ISBN: 978-1-55563-147-5.

[12] H.J. Yin, D.M. Wang, H.Y. Zhong, S.Y. Meng, H.M. Jiang, E.G. Tang, Flow Characteristics of Viscoelastic Polymer Solution in Micro-Pores, SPE EOR Conference at Oil and Gas West Asia, 16-18 April 2012, Muscat, Oman, ISBN: 978-1-61399-199-2

[13] P.G. Saffman, G. Taylor, The Penetration of a Fluid into a Porous Medium or Hele-Shaw Cell Containing a More Viscous Liquid, Proceedings of the Royal Society of London. Series A. Mathematical and Physical Sciences. 245 (1958) $312-329$.

[14] G.M. Homsy, Viscous Fingering in Porous Media, Annual Review of Fluid Mechanics. 19 (1987) 271-311.

[15] P.G. Saffman, Viscous fingering in Hele-Shaw cells, Journal of Fluid Mechanics. 173 (1986) 73-94.

[16] A. Lindner, D. Bonn, E.C. Poiré, M.B. Amar, J. Meunier, Viscous fingering in non-Newtonian fluids, Journal of Fluid Mechanics. 469 (2002) 237-256.

[17] D.V. Boger, A highly elastic constant-viscosity fluid, Journal of Non-Newtonian Fluid Mechanics. 3 (1977) $87-91$.

[18] R.J. Binnington, D.V. Boger, Remarks on non-shear thinning elastic fluids, Polym Eng Sci. 26 (1986) 133138.

[19] V. Gauri, K.W. Koelling, Extensional theology of concentrated poly(ethylene oxide) solutions, Rheologica Acta. 36 (1997) 555-567.

[20] A. Ait-Kadi, P.J. Carreau, G. Chauveteau, Rheological Properties of Partially Hydrolyzed Polyacrylamide Solutions, Journal of Rheology. 31 (1987) 537-561.

[21] J. Stokes, L. Graham, N. Lawson, D. Boger, Swirling flow of viscoelastic fluids. Part 2. Elastic effects, J. Fluid Mech. 429 (2001) 117-153.

[22] C.W. Macosko, Rheology - Principles, Measurements and Applications, (n.d.).

[23] H.P. Sdougos, S.R. Bussolari, C.F. Dewey, Secondary flow and turbulence in a cone-and-plate device, Journal of Fluid Mechanics. 138 (1984) 379-404.

[24] P. Gondret, N. Rakotomalala, M. Rabaud, D. Salin, P. Watzky, Viscous parallel flows in finite aspect ratio Hele-Shaw cell: Analytical and numerical results, Physics of Fluids. 9 (1997) 1841-1843.

[25] F.P. Bretherton, The motion of long bubbles in tubes, Journal of Fluid Mechanics. 10 (1961) 166-188.

[26] P.-G. de Gennes, F. Brochard-Wyart, D. Quéré, Gouttes, bulles, perles et ondes, Belin, 2002. 
